\title{
Evaluation de la pollution métallique des eaux souterraines et de surface dans un environnement minier aurifère à Hiré (Côte d'Ivoire)
}

\author{
Y.H.A. YAPI ${ }^{1,2^{*}}$, B.K. DONGUI ${ }^{1}$, A. TROKOUREY ${ }^{2}$, Y.S.S. BARIMA ${ }^{1}$, \\ Y. ESSIS $^{3}$ et P. ATHEBA ${ }^{2}$ \\ ${ }^{1}$ Université Jean Lorougnon Guédé, Unité de formation et de Recherches en Environnement, \\ BP 150 Daloa, Côte d'Ivoire. \\ ${ }^{2}$ Université Félix Houphouët Boigny, Unité de Formation et Recherche en Sciences des Structures de la \\ Matière et des Technologies (SSMT), Laboratoire de Chimie Physique, 22 BP 582 Abidjan 22, Côte d'Ivoire. \\ ${ }^{3}$ Ecole Normale Supérieure, Laboratoire de Chimie des Eaux, 08 BP 10 Abidjan 08, Côte d'Ivoire. \\ *Auteur correspondant ; E-mail : yyapohermannaristide@gmail.com
}

\section{RESUME}

Cette étude a pour objet l'évaluation du taux de pollution métallique des eaux souterraines et de surface dans un environnement minier. Pour y parvenir, des prélèvements d'eaux ont été effectués au cours de deux campagnes d'échantillonnage (mois décembre 2012 et mois de février 2013) dans la Sous-préfecture de Hiré (centre ouest de la Côte d'Ivoire). Les résultats ont montré des concentrations élevées pour tous les paramètres métalliques dans la majorité des stations. En effet, les teneurs d'arsenic, de cadmium, de mercure, de nickel, de plomb sont largement supérieures aux valeurs limites fixées par l'OMS pour les eaux de consommation et d'irrigation. En outre, elles pourraient constituer des risques sanitaires pour la population de la zone d'étude. Nos résultats serviront de base aux politiques environnementales nationales visant à protéger les populations vivant dans un environnement minier.

(C) 2014 International Formulae Group. All rights reserved.

Mots clés : Pollution, métaux, sols, Hiré, Côte d'Ivoire.

\section{INTRODUCTION}

Depuis les indépendances en 1960, les activités minières, à l'exception des hydrocarbures, ne jouaient pas un rôle prépondérant dans l'économie ivoirienne. Cependant, les importantes découvertes des gisements des quinze dernières années ont amené le gouvernement ivoirien à mettre l'accent sur la politique de développement du secteur minier afin de le redynamiser. Même si le développement minier est jugé par les uns comme un facteur positif au plan des retombés socio-économiques, il est critiqué par d'autres pour ses impacts environnementaux négatifs (Aubertin et al., 2002 ; Ripley et al., 2002 ; Bridge et al., 2004), en particulier dans les régions éloignées, peu développées où vivent des communautés autochtones dont une partie du mode de vie traditionnel dépend de l'intégrité écologique du territoire (Scott, 2002).

En Côte d'Ivoire, la contamination métallique des écosystèmes a attiré l'attention de nombreux chercheurs (Keumean et al., 2013 ; Touré et al., 2010 ; Coulibaly et al., 2009). Elle constitue en effet, l'un des aspects 
de la pollution le plus menaçant pour ces milieux. Par ses effets néfastes, elle pourrait engendrer des situations critiques (perte de toute vie aquatique, intoxication métallique de la population) voire dangereuses affectant parfois l'équilibre écologique de ces écosystèmes (Makhoukh et al., 2011). La pollution métallique constitue un problème d'actualité qui préoccupe toutes les régions soucieuses de maintenir leur patrimoine hydrique à un haut degré de qualité. Cette préoccupation touche la Sous-préfecture de Hiré, située dans le centre ouest de la Côte d'Ivoire où une importante activité minière est menée depuis les années 2000. Cette activité minière se matérialise par plusieurs sites d'exploitations artisanales d'or qui ceinturent la ville de Hiré et d'une mine d'or (la mine de Bonikro) qui se trouve à $10 \mathrm{~km}$ de la ville.

Cette mine d'or est à ciel ouvert où le traitement du minerai se fait par extraction chimique au cyanure et par lixiviation en cuves. Le stockage des résidus miniers se fait dans un grand bassin de décantation fermé par une digue. L'exploitation de la mine d'or de Bonikro et les activités d'orpaillage pourraient avoir des impacts sociaux et environnementaux dans les villages autour de la mine et dans la ville de Hiré. En effet, de nombreuses liaisons chimiques sont possibles au sein des minéraux sulfureux et incluent parfois plusieurs éléments métalliques dans un minéral, par exemple la pyrite $\left(\mathrm{FeS}_{2}\right)$, l'arsénopyrite (FeAsS), la galène $(\mathrm{PbS})$, la cinabre $\mathrm{HgS}$, la sphalérite $\mathrm{ZnS}$. (Miramond et al., 2006). L'accumulation des contaminants métalliques dans les organismes aquatiques présente des effets toxicologiques sur les espèces, les écosystèmes et les risques sanitaires à travers l'ingestion d'espèces comestibles (Pétré, 2008 ; Burnol et al., 2004).

L'objectif de ce travail est de déterminer l'impact des activités aurifères sur la qualité chimique des eaux souterraines et de surface de la Sous-préfecture de Hiré. Pour y parvenir, les éléments traces métalliques de certains points d'eaux de surface et souterraine aux alentours de la mine et dans la ville de Hiré ont été mesuré.

\section{MATERIEL ET METHODES}

Présentation de la zone d'étude

Notre zone d'étude est située entre $06^{\circ} 15^{\prime} 08.6$ et $06^{\circ} 10^{\prime}$ de latitude nord et $05^{\circ} 23^{\prime} 44.8$ et $05^{\circ} 16^{\prime} 32.1$ de longitude ouest. Le climat est de type équatorial avec une pluviométrie moyenne d'environ $1200 \mathrm{~mm}$. L'étude pédologique de la zone d'étude a mis en évidence sept profils de sols appartenant à trois groupes (plinthosol, ferrasol, gleysol) (Atsé, 2007) qui ont été définis par le système taxonomique de la FAO. Tous ces sols résultent d'une altération longue et intense, avec une fraction argileuse composée essentiellement de kaolinite (Atsé, 2007). Les principaux minéraux sulfureux présents sont la pyrite, la galène, la bismuthine, la blende et la chalcopyrite (Sonnendrucker, 1967). Les minéraux accessoires sont le mispickel, la stibine (Sonnendrucker, 1967).

\section{Echantillonnage}

Les prélèvements des échantillons ont été effectués au cours de deux campagnes dans les mois de décembre 2012 et février 2013. Des échantillons d'eau ont été prélevés dans dix-sept stations notées Pn, où $n$ est le numéro de la station. Leurs choix résultent d'un compromis entre les possibilités d'y accéder et les possibilités de rendre compte de la pollution métallique des eaux.

$\mathrm{P}_{1}, \mathrm{P}_{2}, \mathrm{P}_{9}$, et $\mathrm{P}_{16}$ sont des stations où des échantillons d'eau de puits paysans ont été prélevés et qui sont situées dans la ville d'Hiré (Figure 1). $\mathrm{P}_{1}, \mathrm{P}_{2}$ et $\mathrm{P}_{9}$ sont situées à $11 \mathrm{~km}$ de la mine tandis que $\mathrm{P}_{16}$ est située à $10 \mathrm{~km}$ de la mine. 
Les échantillons des stations $\mathrm{P}_{10}, \mathrm{P}_{11}$, $\mathrm{P}_{12}, \mathrm{P}_{13}, \mathrm{P}_{15}$, et $\mathrm{P}_{17}$ proviennent d'eaux de pompes villageoises. Les cinq premières stations sont situées à moins de $5 \mathrm{~km}$ de la mine. La station $\mathrm{P}_{17}$ est située dans la ville d'Hiré et à $11 \mathrm{~km}$ de la mine.

$\mathrm{P}_{3}, \mathrm{P}_{4}, \mathrm{P}_{6}$ et $\mathrm{P}_{14}$ sont des stations où des échantillons d'eau de surface ont été recueillis. Ces stations sont situées dans des bas-fonds où sont présentes des exploitations agricoles de riz et de cultures maraîchères (gombo, salade, choux, etc.). $\mathrm{P}_{3}$ et $\mathrm{P}_{4}$ sont aussi situées dans la ville d'Hiré et à $11 \mathrm{~km}$ de la mine. $\mathrm{P}_{6}$ est proche des sites d'orpaillages, à $12 \mathrm{~km}$ de la mine alors que $\mathrm{P}_{14}$ est à moins d'un $\mathrm{km}$ de la mine, non loin de la décharge de débris de roche ou mort de terrain de la mine. $\mathrm{P}_{14}$ pourrait recevoir les effluents provenant du parc de débris de roche de la mine.

$\mathrm{P}_{5}$ est un effluent liquide provenant des sites d'orpaillage, à $12 \mathrm{~km}$ de la mine et qui se déversent dans la station $\mathrm{P}_{6}$. Les stations $\mathrm{P}_{7}$, et $\mathrm{P}_{8}$ sont situés sur des zones de rejets des eaux d'orpaillage et à $13 \mathrm{~km}$ de la mine.

\section{Mesure des paramètres physiques}

Dans chacun des sites, la température, le $\mathrm{pH}$, la conductivité ont été mesurés in-situ à l'aide d'un multimètre HACH HQ 40 d.

Des échantillons d'eau ont été prélevés à l'aide de bouteille en plastique de 1L, remplies jusqu'à ras-bord et préalablement rincées avec l'eau de la station. Ils ont été ensuite acidifiés sur le terrain avec de l'acide nitrique $\left(\mathrm{HNO}_{3}\right)$, conservés à $4{ }^{\circ} \mathrm{C}$ pour des analyses des paramètres chimiques (arsenic, cadmium, chrome, mercure, sodium, nickel, plomb, zinc) au laboratoire.

\section{Mesure des paramètres chimiques}

Les échantillons d'eau ont été préalablement filtrés à l'aide d'un filtre GFC Wattman 0,45 $\mu \mathrm{m}$, puis analysés par spectroscopie d'émission dont la source est un plasma généré par un couplage inductif (ICPAES). Le rôle du plasma est de casser les liaisons moléculaires pour produire des ions, atomes libres afin qu'ils soient excités.

\section{RESULTATS}

Les mesures in-situ nous ont permis de faire plusieurs constats. Le $\mathrm{pH}$ des échantillons d'eau pour toutes les stations est compris entre 6,5 et 8,5 sauf pour les stations $\mathrm{P}_{14}(6,2), \mathrm{P}_{9}(5,9)$ et $\mathrm{P}_{12}(5,3)$ (Tableau 1). Les sites étudiés ont une température moyenne de $30,3{ }^{\circ} \mathrm{C}$. Cependant, la température des eaux souterraines varie de $28,1{ }^{\circ} \mathrm{C}$ à $32,9{ }^{\circ} \mathrm{C}$ et celle des stations qui reçoivent les effluents miniers $\left(\mathrm{P}_{7}, \mathrm{P}_{8}, \mathrm{P}_{5}, \mathrm{P}_{14}\right)$ ne dépasse pas $29^{\circ} \mathrm{C}$. Les plus fortes conductivités ont été enregistrées pour les stations $\mathrm{P}_{1}(1060 \mu \mathrm{S} / \mathrm{cm})$, $\mathrm{P}_{2}(2660 \mu \mathrm{S} / \mathrm{cm}), \mathrm{P}_{3}(1930 \mu \mathrm{S} / \mathrm{cm})$ et $\mathrm{P}_{6}(1890$ $\mu \mathrm{S} / \mathrm{cm})$. Pour toutes les stations d'eaux souterraines et de surface proches de la mine (moins de $5 \mathrm{~km}$ ), on note une diminution significative de la conductivité.

Les analyses faites au laboratoire permettent de mettre en évidence trois groupes de paramètres métalliques.

Dans le premier groupe, le chrome présente des teneurs très faibles pour toutes les stations, sauf pour la station $\mathrm{P}_{14}$ qui présente une teneur significative $(81 \mu \mathrm{g} / \mathrm{L})$.

L'arsenic, le cadmium, le mercure, le plomb et le zinc font partie du second groupe. En effet, ces métaux ont une concentration inférieure à $1 \mathrm{mg} / \mathrm{L}$ dans la majorité des sites étudiés. Les résultats montrent que ceux-ci sont présents dans toutes les stations à l'exception du plomb qui est quasiment inexistant dans les stations $\mathrm{P}_{2}(1,5 \mu \mathrm{g} / \mathrm{l}), \mathrm{P}_{8}$ $(0,5 \mu \mathrm{g} / \mathrm{l})$ et $\mathrm{P}_{9}(0 \mu \mathrm{g} / \mathrm{l})$. Pour l'arsenic, le plomb et le zinc, les eaux souterraines proches de la mine (moins de $5 \mathrm{~km}$ ) ont des teneurs élevées par rapport aux autres sites avec une concentration maximale en arsenic $(186 \mu \mathrm{g} / \mathrm{l})$, 
plomb $(255,5 \mu \mathrm{g} / \mathrm{l})$ et zinc $(750 \mu \mathrm{g} / \mathrm{l})$ pour la même station $\mathrm{P}_{13}$. Par contre, les eaux de surface ont les plus fortes concentrations en cadmium et en mercure avec des concentrations maximales pour les stations $\mathrm{P}_{3}$ (463 $\mu \mathrm{g} / \mathrm{l})$ et $\mathrm{P}_{4}(541 \mu \mathrm{g} / \mathrm{l})$.

Le dernier groupe comprend le sodium et le nickel dont les concentrations déterminées sont supérieures à $1 \mathrm{mg} / \mathrm{l}$ pour la majorité des sites étudiés. Concernant le sodium, les stations situées dans la ville de
Hiré et proches des sites d'orpaillage ont les valeurs les plus élevées en sodium supérieures $40 \mathrm{mg} / \mathrm{l}$. On note cependant une diminution significative des teneurs en sodium pour toutes les stations d'eaux souterraines et de surface proches de la mine (moins de 5 $\mathrm{km}$ ), alors que le nickel a une distribution inverse à celle du sodium avec les plus fortes concentrations pour les stations $\mathrm{P}_{10}(2,6 \mathrm{mg} / \mathrm{l})$, $\mathrm{P}_{11}(3,3 \mathrm{mg} / \mathrm{l}), \mathrm{P}_{13}(2,0 \mathrm{mg} / \mathrm{l})$ et $\mathrm{P}_{14}(2,4 \mathrm{mg} / \mathrm{l})$ proches de la mine.

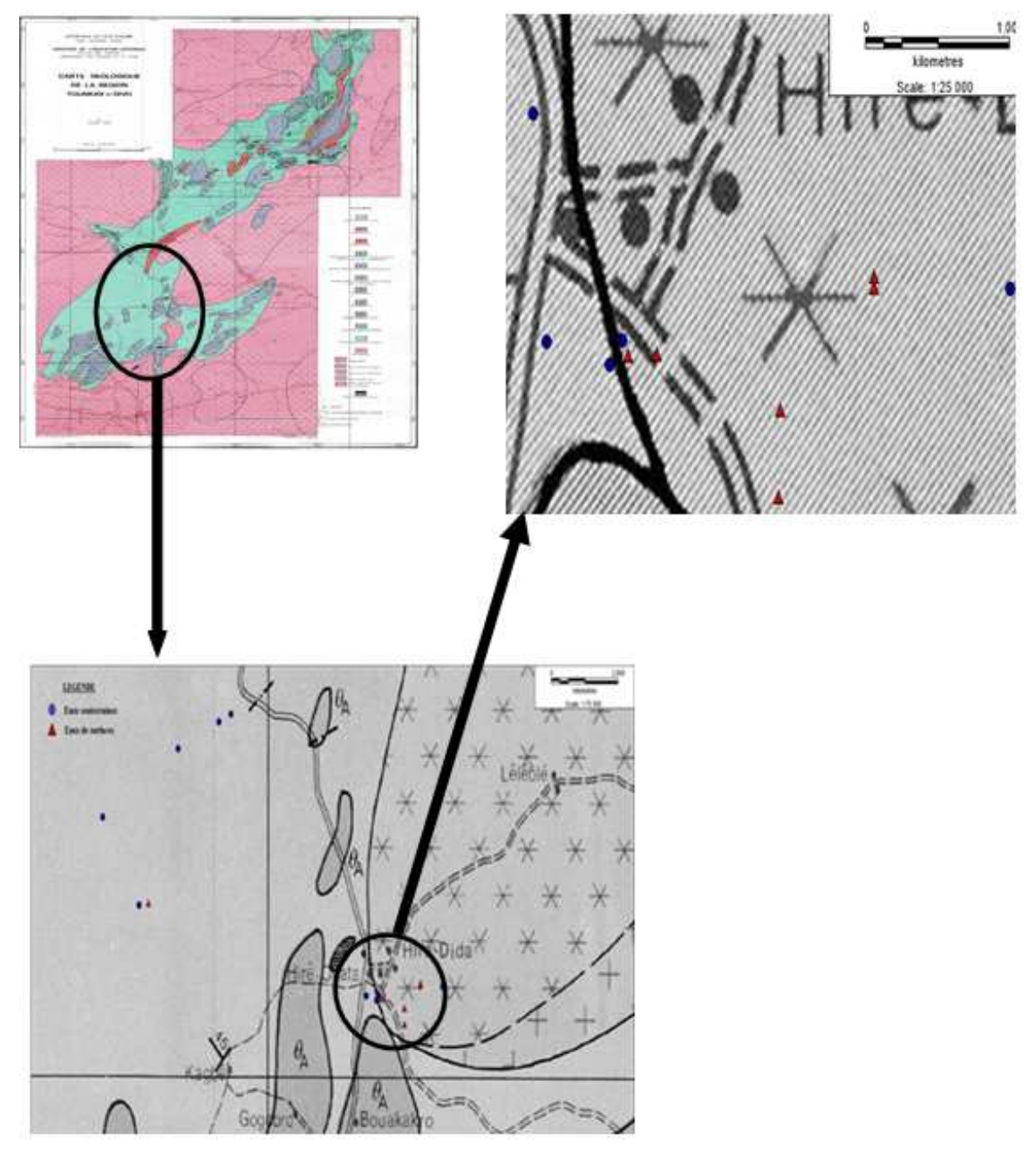

Figure 1 : Situation géographique des différentes stations de prélèvements et carte géologique du secteur d'Hiré. (Direction de la géologie, 1982). 
Tableau 1 : Résultats des mesures des paramètres physiques des différentes stations.

\begin{tabular}{lccc}
\hline Nom de la station & Température $\left({ }^{\circ} \mathbf{C}\right)$ & $\mathbf{p H}$ & Conductivité $(\boldsymbol{\mu} \mathbf{S} / \mathbf{c m})$ \\
\hline P1 & 31,5 & 7,4 & 1060 \\
P2 & 31,1 & 7,8 & 2660 \\
P3 & 31,3 & 7,8 & 1930 \\
P4 & 32,8 & 7,9 & 590 \\
P5 & 29 & 7,0 & 190 \\
P6 & 32,8 & 7,3 & 1890 \\
P7 & 28,4 & 7,8 & 600 \\
P8 & 29,0 & 7,5 & 620 \\
P9 & 28,1 & 5,9 & 280 \\
P10 & 32,9 & 7,8 & 410 \\
P11 & 28,9 & 6,5 & 340 \\
P12 & 30,1 & 5,3 & 230 \\
P13 & 30,3 & 7,9 & 290 \\
P14 & 28,6 & 6,2 & 60 \\
P15 & 32,0 & 7,7 & 430 \\
P16 & 29,5 & 6,7 & 610 \\
P17 & 30 & 7,5 & 240 \\
Normes de l'OMS & $20-25$ & $6,5-$ & 250 \\
& & 9,5 & \\
\hline
\end{tabular}

Tableau 2 : Concentration moyenne de paramètres chimiques dans différentes stations.

\begin{tabular}{|c|c|c|c|c|c|c|c|c|}
\hline $\begin{array}{l}\text { Nom de la } \\
\text { station }\end{array}$ & $\begin{array}{c}\mathbf{A s} \\
(\mu \mathrm{g} / \mathrm{l})\end{array}$ & $\begin{array}{c}\mathbf{C d} \\
(\mu \mathrm{g} / \mathrm{l})\end{array}$ & $\begin{array}{c}\mathrm{Cr} \\
(\mu \mathrm{g} / \mathrm{l})\end{array}$ & $\underset{(\mu \mathrm{g} / \mathrm{l})}{\mathbf{H g}}$ & $\begin{array}{c}\mathrm{Na} \\
(\mathrm{mg} / \mathrm{l})\end{array}$ & $\begin{array}{c}\mathbf{N i} \\
(\mathrm{mg} / \mathrm{l})\end{array}$ & $\begin{array}{c}\mathrm{Pb} \\
(\mu \mathrm{g} / \mathrm{l})\end{array}$ & $\begin{array}{c}\mathbf{Z n} \\
(\mu \mathrm{g} / \mathrm{l})\end{array}$ \\
\hline $\mathrm{P}_{1}$ & 54,5 & 185 & 13 & 25 & 45,50 & 1,4 & 73 & 416 \\
\hline $\mathrm{P}_{2}$ & 63 & 202 & 26 & 286 & 174,5 & 1,9 & 1,5 & 92 \\
\hline $\mathrm{P}_{3}$ & 152 & 463 & 31 & 328 & 79,6 & 1,2 & 20,5 & 266 \\
\hline $\mathrm{P}_{4}$ & 48,5 & 179 & 2 & 541 & 20,3 & 1,3 & 170 & 318 \\
\hline $\mathrm{P}_{5}$ & 135 & 267 & 11 & 156 & 44,6 & 1,4 & 49 & 448 \\
\hline $\mathrm{P}_{6}$ & 75 & 209 & 7 & 280 & 72,3 & 2,1 & 55,5 & 258 \\
\hline $\mathrm{P}_{7}$ & 162,5 & 380 & 8 & 360 & 54,8 & 1,3 & 94 & 227 \\
\hline $\mathrm{P}_{8}$ & 129 & 365 & 1 & 261 & 53,5 & 1,2 & 0,5 & 479 \\
\hline $\mathrm{P}_{9}$ & 47,5 & 159 & 3 & 324 & 31 & 0,6 & 0 & 253 \\
\hline $\mathrm{P}_{10}$ & 109,5 & 78 & 5 & 17 & 17,4 & 2,6 & 101,5 & 467 \\
\hline $\mathrm{P}_{11}$ & 113 & 86 & 1 & 54 & 22,7 & 3,3 & 97,5 & 563 \\
\hline $\mathrm{P}_{12}$ & 183,5 & 291 & 13 & 452 & 21,7 & 0,5 & 47,5 & 591 \\
\hline$P_{13}$ & 186,5 & 107 & 7 & 44 & 13,8 & 2,0 & 255,5 & 750 \\
\hline $\mathrm{P}_{14}$ & 68 & 334 & 81 & 745 & 6,7 & 2,4 & 52,5 & 257 \\
\hline $\mathrm{P}_{15}$ & 110,5 & 78 & 3 & 52 & 13,2 & 2,6 & 99 & 610 \\
\hline $\mathrm{P}_{16}$ & 136 & 385 & 11 & 665 & 41,6 & 820 & 41 & 477 \\
\hline $\mathrm{P}_{17}$ & 116,5 & 170 & 5 & 169 & 12,6 & 2,1 & 61,5 & 515 \\
\hline $\begin{array}{l}\text { Normes de } \\
\text { l'OMS }\end{array}$ & 10 & 1 & 50 & 6 & 200 & 220 & 10 & $3(\mathrm{mg} / \mathrm{l})$ \\
\hline
\end{tabular}




\section{DISCUSSION}

Nos travaux ont montré que la température moyenne des eaux des différentes stations est de $30,3{ }^{\circ} \mathrm{C}$. Entre 10 et $30{ }^{\circ} \mathrm{C}$, la température n'a qu'un effet négligeable sur la mobilité des métaux (Serpeaud et al., 1994). Mais dans un environnement minier, une température moyenne de $30,3{ }^{\circ} \mathrm{C}$ pourrait influencer le drainage minier acide (DMA) qui constitue le problème environnemental le plus important de l'industrie extractive (Dudka et al., 1997). En effet, elle pourrait accélérer les réactions d'oxydations des minéraux sulfureux libérant ainsi les métaux présents dans ces minéraux. Ainsi, la réaction d'oxydation de la pyrite contribuant à la formation du DMA et la libération des métaux s'expriment sous la forme suivante :

$2 \mathrm{FeS}_{2}+7 \mathrm{O}_{2}+2 \mathrm{H}_{2} \mathrm{O} \rightarrow 2 \mathrm{Fe}^{2+}+$ $4 \mathrm{SO}_{4}{ }^{2-}+4 \mathrm{H}^{+}$

Dans la majorité des sites étudiés, le $\mathrm{pH}$ des eaux souterraines et de surface, compris entre 6,5 et 8,5, est contrôlé par les activités biologiques (photosynthèse et respiration) mais aussi par le pouvoir tampon des eaux, en particulier $\mathrm{CO}_{3}{ }^{2-}, \mathrm{HCO}_{3}{ }^{-}$et $\mathrm{CO}_{2}$ (Taouil., 2012 ; Makhoukh et al., 2011 ; Sigg et al., 2006). Par contre, l'acidité des stations $P_{9}$ $(5,9) \mathrm{P}_{12}(5,3) \mathrm{P}_{14}(6,2)$ pourrait être due à la précipitation des hydroxydes qui génère une acidité du milieu et consomme les métaux (Miaramond et al., 2006) ; ce qui explique la faible conductivité mesurée pour ces stations $\begin{array}{lllllll}\mathrm{P}_{9} & (280 & \mu \mathrm{S} / \mathrm{cm}) & \mathrm{P}_{12} & (230 & \mu \mathrm{S} / \mathrm{cm}) & \mathrm{P}_{14}\end{array}$ $\mu \mathrm{S} / \mathrm{cm})$. En outre, nos résultats ont montré que la station $\mathrm{P}_{14}$ présente des concentrations maximales en chrome $(81 \mu \mathrm{g} / \mathrm{l})$ et en mercure (745 $\mu \mathrm{g} / \mathrm{l})$ qui pourraient s'expliquer par la contamination de cette eau de surface par les eaux d'infiltration provenant du parc de débris de roche de la mine (Atsé, 2007). Il faut rappeler que cette station est à moins d'un km de la mine et proche du parc à résidus de stériles.

Les faibles teneurs en chrome déterminées pour la majorité des échantillons pourraient être dues au pH. En effet, la mobilité du $\mathrm{Cr}(\mathrm{III})$, en milieu aqueux légèrement acide à basique, est limitée à cause de la faible solubilité de $\mathrm{Cr}(\mathrm{OH})_{3}(\mathrm{~s})$ et $(\mathrm{Fe}, \mathrm{Cr})(\mathrm{OH})_{3}$ (s) (Lespagnol, 2003 ; RaÏ et al., 1987 ; Sass et RaII, 1987). En revanche, la mobilité du $\mathrm{Cr}(\mathrm{VI})$ est guidée par les réactions d'adsorption et de précipitation (N'guessan, 2008 ; Lespagnol, 2003).

Les eaux d'infiltration du parc à résidus et du bassin de décantation pourraient être aussi la cause de fortes concentrations des eaux souterraines proches de la mine d'or (moins de $5 \mathrm{~km})$ en $\operatorname{arsenic}\left(\mathrm{P}_{12}, 183,5 \mu \mathrm{g} / \mathrm{l}\right.$; $\left.\mathrm{P}_{13}, 186,5 \mu \mathrm{g} / \mathrm{L}\right)$, cadmium $\left(\mathrm{P}_{12}, 291 \mu \mathrm{g} / \mathrm{l}\right)$, mercure $\left(\mathrm{P}_{12}, 452 \mu \mathrm{g} / \mathrm{l}\right)$, plomb $\left(\mathrm{P}_{13}, 255,5\right.$ $\mu \mathrm{g} / \mathrm{l}$ ) (Atsé, 2007). Le site d'étude étant une zone aurifère, l'oxydation des minéraux sulfureux (Miramond, 2006) des sédiments par l'intrusion de l'oxygène dans ces nappes libèrent les métaux et pourraient contaminer les eaux souterraines selon le mécanisme des réactions ci-dessus.

Par ailleurs, les eaux souterraines situées dans la ville de Hiré ont toutes des concentrations relativement élevées pour tous les paramètres étudiés, d'où la forte conductivité mesurée pour les stations $\mathrm{P}_{1}$ $(1060 \mu \mathrm{S} / \mathrm{cm})$ et $\mathrm{P}_{2}(2660 \mu \mathrm{S} / \mathrm{cm})$. Nous pouvons l'expliquer par le mauvais entretien des puits paysans dont l'eau favorise la dissolution des métaux présents dans les roches et les sédiments. Le phénomène de lixiviation des roches et sédiments serait l'une des causes de la forte présence du sodium, cadmium, mercure, nickel dans les eaux de surface. En effet, la plupart des roches et des sédiments contiennent des composés du sodium, du mercure (HgS) (Miramond et al., 2006) que l'on rencontre dans la plupart des gisements aurifères, du cadmium, du nickel (N'guessan, 2008). En plus, la ville de Hiré n'ayant pas de système de canalisation, il faudrait ajouter le déversement des eaux usées qui serait responsable des fortes teneurs en sodium et zinc (Taouil, 2012). Les activités d'exploitations artisanales de l'or seraient aussi responsables des fortes concentrations 
de ces métaux dans les eaux de surfaces, puisque les effluents chargés de métaux et la lixiviation des résidus solides provenant de ces activités sont déversés directement dans l'environnement. La station $\mathrm{P}_{5}$ met en évidence cet enjeu environnemental. Il faut rappeler que $\mathrm{P}_{5}$ est un effluent liquide provenant d'un site d'orpaillage.

Par ailleurs, le pH joue un rôle important dans l'adsorption des métaux. En effet, un $\mathrm{pH}$ alcalin limite le passage des métaux de la phase solide à la phase aqueuse (Thornton, 1996). Les stations dont le $\mathrm{pH}$ varie entre 7,04 et 7,89 , induisent systématiquement une augmentation du taux d'adsorption des éléments traces dans les sédiments superficiels. Mais le piégeage des éléments traces n'est pas forcement définitif. Les risques de remobilisation, de biodisponibilité et donc de toxicité sont à craindre. Ils constituent, en effet, un danger permanent pour tout l'écosystème aquatique lorsque les conditions physico-chimiques le permettent (François, 2002), notamment dans la chaîne trophique (Fadil et al., 1997).

Nos résultats ont aussi montré que les concentrations en arsenic, cadmium, mercure, nickel sont largement supérieures aux valeurs limites fixées par l'OMS (Tableau 2) pour les eaux de consommation. Pour le plomb, seules les stations $\mathrm{P}_{2}, \mathrm{P}_{9}$ et $\mathrm{P}_{8}$ auraient des eaux de qualité acceptable pour la consommation puisque ayant des teneurs inférieures à la valeur limite fixée par l'OMS (Tableau 2). Ces faibles teneurs pourraient être dues au contexte géologique propre à ces différentes stations. Concernant le chrome, toutes les stations ont des eaux acceptables à la consommation, à l'exception de la station $\mathrm{P}_{14}$ dont la concentration déterminée est supérieure à la valeur limite fixée par l'OMS. Par contre, certains métaux, dont le sodium et le zinc, ont des concentrations pour toutes les stations inférieures aux valeurs limites fixées par l'OMS. Selon ces normes de qualité, les eaux des différentes stations étudiées sont acceptables pour la consommation.
La qualité d'une eau dépendant aussi des paramètres physico-chimiques, alors, on pourrait affirmer que les eaux des différentes stations étudiées sont non potables pour la consommation humaine.

Conscients des capacités d'adsorption des métaux par les végétaux, il y a des risques de contamination métallique de la population de Hiré via la consommation des produits de cultures maraîchères. En effet, les stations $\mathrm{P}_{3}$, $\mathrm{P}_{4}, \mathrm{P}_{6}$ et $\mathrm{P}_{14}, \quad$ situées dans des zones d'exploitation agricole de riz et de cultures maraîchères, présentent des concentrations en arsenic, cadmium et mercure supérieures aux valeurs limites fixées par l'OMS pour des eaux destinées à l'irrigation.

\section{Conclusion}

Les résultats obtenus dans ce travail permettent de procéder à une évaluation de la pollution métallique de l'eau environnante des sites d'exploitations artisanales et industrielle de l'or dans la Sous-préfecture de Hiré. Ces résultats montrent que les concentrations de $\mathrm{As}, \mathrm{Cd}, \mathrm{Hg}, \mathrm{Ni}$ et $\mathrm{Pb}$ sont relativement élevées dans toutes les stations étudiées. En dehors du $\mathrm{Na}$ et $\mathrm{Zn}$, les autres métaux ont des teneurs qui dépassent largement les normes fixées par l'OMS de potabilité pour toutes les stations occasionnant ainsi la détérioration de la qualité de ces eaux. Par conséquent, leur utilisation pourrait avoir des impacts négatifs sur la santé de la population. Par ailleurs, il y a pollution naturelle de la part des roches.

$\mathrm{Au}$ total, les eaux de boissons consommées dans la zone d'étude ne sont pas de bonne qualité. Les eaux de surface non plus ne devraient pas être utilisées pour l'irrigation agricole.

Dans la majorité des sites étudiés, le pH étant sensiblement basique, il faut craindre une remobilisation des métaux lors des changements physico-chimiques. 


\section{REFERENCES}

Atse ARM. 2007. Etude d'impact environnemental $\mathrm{du}$ projet aurifère de Bonikro en Côte d'Ivoire. Institut de l'Energie et de l'Environnement de la Francophonie, Fiche technique moged.

Aubertin M, Bussière B, Bernier L. 2002. Environnement et Gestion des Rejets Miniers (Manuel sur cédérom). Presses Internationales Polytechnique : Montréal.

Brigde G. 2004. Contested terrain: Miningand the environment. Annual Review of the Environmnent and Resources, 29: 205-209.

Burnol A, Blanc P, Guyonnet D. 2004. Recommandations pour la Modélisation des Transferts Métaliques dans les Eaux Souterraines. Rapport final. BRGM/RP52910, France.

Coulibaly AS, Mondé S, Wognin VA, Aka K. 2009. Analyse des éléments traces métalliques (ETM) dans les baies estuariennes d'Abidjan en Côte d'ivoire. Afrique Sciences, 05(3): 77-96.

Dudka S, Adriano DC. 1997. Environmental of impact metal ore mining and prosessing: A Review, Journal of Environmental Quality, 26: 590-602

El hachimi ML, El hanbali M, Fekhaoui M, Bouabdli A, El Founti L, Saidi N. 2005. Impact d'un site minier abandonné sur l'environnement : cas de la mine de Zaida (haute Moulouya, Maroc). Bulletin de l'Institut Scientifique, Rabat, Section Sciences de la Terre, 27: 93-100.

Fadil F, Maarouf A, ZAID A. 1997. Utilisation de Gammarus gauthieri pour tester la toxicité des sédiments des eaux douces. Limnol, 32: 73-78.

François M, Li D, Dubourguier HC, Douay F. 2002. Facteurs déterminants de la mobilité $(\mathrm{Pb}, \mathrm{Cu}, \mathrm{Zn})$ dans les sols contaminés autour de deux usines métallurgiques du Nord de la France, Journées Nationales de l'étude des sols, du 22-24 octobre, 2002, Orléans.
Keumean KN, Bamba SB, Soro G, Soro N, Métongo BS et Biémi J. 2013. Concentration en métaux lourds des sediments de l'estuaire du fleuve comoé. Journal of Applied Biosciences, 61: 45304539.

Lespagnol G. 2003. Lixiviation du chrome, du cuivre et de l'arsenic (CCA) à partir de sols contaminés sur des sites de traitement du bois. Thèse de Doctorat, Ecole normale supérieures des mines de Saint-Etienne. Université Jean Monnet, Saint-Etienne, p.182.

Makhoukh M, Sbaa M, Berrahou A, Vanclooster M, 2011. Contribution à l'étude de l'impact d'un site minier abandonné dans la haute Moulouya sur la qualité de l'Oued Moulouya, Maroc. Afrique Sciences, 07(3): 34-48.

Miramond N, Miau D, Brochard F. 2006. Diagnostic du phénomène Drainage Minier Acide sur des mines d'or primaire en Guyane françaises: Evaluation des risques associés, Rapport, GEM ImpactDIREN, Guyane française.

N'guessan YM. 2008. Dynamisme des éléments traces sans les eaux de surface des bassins versants agricoles de Cascogne. Thèse de Doctorat, Institut national polytechnique de Toulouse (INPT), Université de Toulouse, Toulouse, p.253.

Pétré MA, 2008. Etude de la spéciation des métaux dissous en seine, relation avec le $\mathrm{pH}$ et la matière organique dissoute. Master 2, Université Pierre et Marie Curie, Ecole des mines de Paris et Ecole National du Génie Rural et des Eaux et Forêts, Paris, p.46.

Rai D, Sass BM, Moore DA. 1987. Chromium(III) hydrolysis constants and solubility of chromium(III) hydroxide. Inorganic Chemistry, 26: 345-349.

Ripley EA, Redman RE, Crowder AA. 1996. Environmental Effects of Mining. Saint Lucie Press: Delray Beach, Floride. 
Sass MB, Rai D. 1987. Solubility of amorphous chromium (III)-iron(III) hydroxide solid solutions. Inorganic Chemistry, 26: 2228-2232.

Scott CH (dir). 2002. Aboriginal Autonomy and Development in Northen Quebec and Labrador. University of British Columbia Press: Vancouver and Toronto.

Serpeaud B, Al-Shukry R, Casteigneau M, Matejka G. 1994. Adsorption des métaux $(\mathrm{Cu}, \mathrm{Zn}, \mathrm{Cd}$ et $\mathrm{Pb})$ par les sédiments superficiels d'un cours d'eau, Rôle du $\mathrm{pH}$, de la température et de la composition des sédiments. Revues des Sciences de l'Eau, 7: 343-365.

Sigg L, Behra P, Stumm W, 2006. Chimie des Milieux Aquatiques, Chimie des Eaux Naturelles et des Interfaces dans l'Environnement (4 ${ }^{\mathrm{e}}$ edn). Dunod: Paris.

Sonnendrucker P. 1967. Etude de la synthèse sur l'or en Côte d'Ivoire: La région aurifère du pays Dida. Rapport 200. SODEMI.

Taouil H, Ahmed IS, Hajjaji N, Srhiri A. 2012. Evaluation de la pollution métallique : $\mathrm{Mn}, \mathrm{Fe}, \mathrm{Zn}$, $\mathrm{Co}$ et $\mathrm{Cr}$ des eaux de l'oued tislit-talsin. Maroc. ScienceLib Editions Mersenne, 4: 1-11.

Thornton I. 1996. Risk assessment related to metals: the role of the geochemist. Report of the International Wordshop on Risk Assessment of Metals and their Inorganic Compound, International Council on Metals and the Environment, Angers, France.

Touré N, Yao KA, Alui KA, Guety TP. 2010. Evaluation en éléments majeurs et trace métalliques d'un environnement de production agricole dans la vallée du Niéki au Sud-Est de Côte d'Ivoire. Journal of Applied Biosciences, 34: 21342144. 